\title{
Mélange of Lymphoepithelial Lesions of Salivary Glands from a Tertiary Care Center of North East India: Diagnostic Conundrums
}

\author{
Zachariah Chowdhury ${ }^{1}$ Vandana Raphael ${ }^{2}$ Yookarin Khonglah ${ }^{2}$ Jaya Mishra² \\ Evarisalin Marbaniang ${ }^{2}$ Biswajit Dey²
}

1Department of Pathology, Mahamana Pandit Madan Mohan Malaviya Cancer Centre and Homi Bhabha Cancer Hospital (Tata Memorial Hospital), Varanasi, Uttar Pradesh, India

2Department of Pathology, North Eastern Indira Gandhi Regional Institute of Health \& Medical Sciences, Shillong, Meghalaya, India

\begin{abstract}
Address for correspondence Zachariah Chowdhury, MBBS, MD, C/O Dr G.M.S. Chowdhury, Red Cross Road, Dibrugarh, Assam, 786001, India (e-mail: zachtmhvaranasi@gmail.com).
\end{abstract}

J Lab Physicians 2021;13:338-345.

\begin{abstract}
Background Lymphocytic infiltrates of the major salivary glands are involved in a spectrum of diseases that range from reactive to benign and malignant neoplasms. Occasionally, these pathologic entities present difficulties in the clinical and pathological diagnosis.

Aim and Objective The aim of this study was to highlight the importance of meticulous cytopathological and histopathological examination (HPE) in solving the diagnostic challenges encountered in the analysis of these salivary gland lesions.

Materials and Methods A retrospective analysis of salivary gland lesions was undertaken over a period of 5 years from 2013 to 2018 in the Department of Pathology at our institute. Salivary gland pathologies diagnosed either as chronic sialadenitis or reactive/benign/malignant lymphoepithelial lesions on fine-needle aspiration cytology (FNAC) and as lymphoepithelial carcinoma (LEC) were included in this study.

Results A total of 86 cases of salivary gland lesions diagnosed as mentioned above were found during this period. Out of the 86 cases, 16 were subjected to HPE. Biopsy was not warranted in most of the cases diagnosed as chronic sialadenitis. HPE was con-

Keywords

- histopathology

- immunohistochemistry

- lymphoepithelial

- salivary glands

- sialadenitis cordant with the FNAC diagnoses in 13 out of the 16 cases (81.3\%), with a single case misinterpreted as LEC on FNAC.

Conclusion Benign and malignant lymphoepithelial lesions of salivary glands may sometimes be difficult to differentiate not only from one another on FNAC but also from other malignant lesions. FNAC is an effective tool for the diagnosis of nonneoplastic lesions, but in cases of benign lymphoepithelial lesions in the absence of salivary acini, biopsy is advisable.
\end{abstract}

\section{Introduction}

Salivary glands give rise to more than 30 histologically distinct benign and malignant tumors, constituting less than $2 \%$ of all tumors in humans and $3 \%$ of all head and

published online

July 12, 2021
DOI https://doi.org/

$10.1055 / \mathrm{s}-0041-1731973$

ISSN 0974-2727 neck tumors. The most common site is the parotid gland comprising $80 \%$ of cases, and approximately $80 \%$ are benign and $20 \%$ are malignant. Among them are lymphoepithelial lesions (LELs), rare lesions of the salivary glands, characterized by lymphocytic infiltration associated with an epithelial

(c) 2021. The Indian Association of Laboratory Physicians.

This is an open access article published by Thieme under the terms of the Creative Commons Attribution-NonDerivative-NonCommercial-License, permitting copying and reproduction so long as the original work is given appropriate credit. Contents may not be used for commercial purposes, or adapted, remixed, transformed or built upon. (https://creativecommons.org/licenses/by-nc-nd/4.0/).

Thieme Medical and Scientific Publishers Pvt. Ltd. A-12, 2nd Floor, Sector 2, Noida-201301 UP, India 
proliferation. ${ }^{1}$ LELs of salivary gland encompass a heterogeneous group of diseases that include benign reactive lesions and malignant neoplasms. These pathologic entities sometimes pose as diagnostic dilemmas.

\section{Aim and Objective}

The study was undertaken to highlight the importance of meticulous cytopathological and histopathological examination (HPE) in solving the diagnostic challenges encountered in the analysis of these salivary gland lesions.

\section{Materials and Methods}

A retrospective analysis of salivary gland lesions was undertaken over a period of 5 years from 2014 to 2019 in the department of pathology at our institute following all the guidelines of the institutional ethics committee.

\section{Inclusion Criteria}

- Salivary gland pathologies diagnosed either as chronic sialadenitis or reactive/benign/malignant LELs on cytopathological examination were included in this study.

- Salivary gland lesions diagnosed as poorly differentiated malignancy/carcinoma/metastatic carcinoma on cytopathological examination, which turned out to be lymphoepithelial carcinoma (LEC) on HPE.

\section{Exclusion Criteria}

- Other salivary gland pathologies.

- Details of age, gender, and other relevant clinical information were collected from the medical records. May-Grunwald Giemsa stained fine-needle aspiration cytology (FNAC) slides and corresponding Hematoxylin and Eosin stained slides sectioned from formalin fixed paraffin embedded tissues and immunohistochemistry (IHC) slides were retrieved from the archives in the department of pathology and evaluated.

\section{Results}

A total of 86 cases of salivary gland lesions diagnosed as mentioned above on FNAC fulfilled the inclusion and exclusion criteria. Males slightly outnumbered females, ratio being 1.4:1. Age range was very wide, from 1 to 95 years. Out of the 86 cases, 16 were subjected to HPE. Biopsy was not necessary in most of the cases diagnosed as chronic sialadenitis. The submandibular gland was the predominant site of involvement with 58 of these cases (67.44\%), followed by 23 cases of the parotid (26.74\%); three cases involved both the parotid and the submandibular gland (3.49\%), while the minor salivary glands were implicated in two cases $(2.33 \%$; - Table 1). The diagnoses encountered were chronic sialadenitis in 72 cases (83.7\%), granulomatous inflammation in 03 cases (3.5\%), benign lymphoepithelial lesion (BLEL) in 03 cases $(3.5 \%)$, reactive inflammatory lesion in 02 cases (2.3\%), and malignant entities in 06 cases (7\%), thus benign entities totaling 80 and malignant 6. Chronic sialadenitis predominantly affected the submandibular gland, while the other benign/reactive lesions involved the parotid more. Both the major and the minor salivary glands were affected by the malignant entities. Correlation of the cytopathologic diagnosis with HPE is shown in - Table 2. Out of the five cases of chronic sialadenitis diagnosed as such on FNAC for which biopsy was done, $60 \%$ correlation was observed with the HPE diagnosis, that is, three cases, while the remaining two cases turned out to be benign salivary gland neoplasms on HPE, namely Warthin tumor and myoepithelioma. There was no discrepancy in the diagnosis of BLEL/lymphoepithelial sialadenitis between FNAC and HPE (-Fig. 1), whereas a lymphoepithelial cyst in the parotid had been rendered a cytopathologic diagnosis of reactive inflammatory lesion.

FNAC proved to be a competent tool for the malignant LELs, as none of them were missed (-Table 3). However, LEC, the histopathologic diagnosis in these cases, was mostly not categorically stated on cytopathologic analysis. FNAC claimed these cases to be one of poorly differentiated malignancy/poorly differentiated carcinoma/metastatic carcinoma, composed of sheets of large polygonal to round cells having high nucleocytoplasmic ratio, round to oval nuclei, coarse chromatin, prominent nucleoli, and scant cytoplasm ( - Fig. 2); the HPE divulged a tumor exhibiting total replacement and destruction of salivary gland architecture by lymphoid cells along with presence of LELs and aggregates of poorly differentiated large polygonal epithelial cells with round to oval vesicular nuclei, prominent nucleoli, and scant cytoplasm (-Fig. 3). Nevertheless, a lone discordance between a benign and a malignant diagnosis was noted, which

Table 1 Diagnosis vis-à-vis site

\begin{tabular}{|l|l|l|l|l|l|}
\hline Sr. No. & Diagnosis & Parotid (P) & Submandibular (SM) & Minor salivary glands & Both P + M \\
\hline 1. & Chronic sialadenitis & 15 & 55 & 01 & 01 \\
\hline 2. & Granulomatous & 02 & 01 & 0 & 0 \\
\hline 3. & BLEL/LES & 02 & 0 & 0 & 01 \\
\hline 4. & Reactive inflammation & 02 & 0 & 0 & 0 \\
\hline 5. & Malignant & 02 & 02 & 01 & 01 \\
\hline 6. & Total & 23 & 58 & 02 & 03 \\
\hline
\end{tabular}

Abbreviations: BLEL, benign lymphoepithelial lesion; LES, lymphoepithelial sialadenitis. 
Table 2 Correlation of cytopathologic diagnosis with HPE

\begin{tabular}{|l|l|l|l|l|l|l|l|}
\hline SI. no. & Diagnosis (FNAC) & $\begin{array}{l}\text { No. of } \\
\text { cases }\end{array}$ & $\begin{array}{l}\text { HPE } \\
\text { done for }\end{array}$ & $\begin{array}{l}\text { Cor-related } \\
\text { with HPE }\end{array}$ & Percentage (\%) & $\begin{array}{l}\text { Not correlated } \\
\text { with HPE }\end{array}$ & Percentage (\%) \\
\hline 1. & Chronic sialadenitis & 72 & 05 & 03 & 60 & 02 & 40 \\
\hline 2. & Granulomatous & 03 & - & - & - & - & - \\
\hline 3. & BLEL/LES & 03 & 03 & 03 & 100 & - & - \\
\hline 4. & Reactive & 02 & 02 & 01 & 50 & 01 & 50 \\
\hline 5. & Malignant & 06 & 06 & 05 & 83.3 & 01 & 16.7 \\
\hline 6. & Total & 86 & 16 & 12 & 75 & 04 & 25 \\
\hline
\end{tabular}

Abbreviations: BLEL, benign lymphoepithelial lesion; HPE, histopathological examination; LES, lymphoepithelial sialadenitis.

Table 3 Correlation of malignant LELs on FNAC with HPE

\begin{tabular}{|l|l|l|l|}
\hline SI. no. & Site & Diagnosis on FNAC & Diagnosis on HPE \\
\hline 1. & Submandibular gland & Poorly differentiated malignancy & LEC \\
\hline 2. & Submandibular gland & Poorly differentiated malignancy & LEC \\
\hline 3. & Parotid gland & Poorly differentiated carcinoma & LEC \\
\hline 4. & Minor salivary glands & Metastatic PDC/LEC & LEC \\
\hline 5. & Parotid gland & Metastatic PDC/LEC/PDC & LEC \\
\hline 6. & Parotid and submandibular glands & LEC & Granulomatous lymphoepithelial sialadenitis \\
\hline
\end{tabular}

Abbreviations: FNAC, fine-needle aspiration cytology; HPE, histopathological examination; LEC, lymphoepithelial carcinoma; LELs, lymphoepithelial lesions; PDC, poorly differentiated carcinoma.
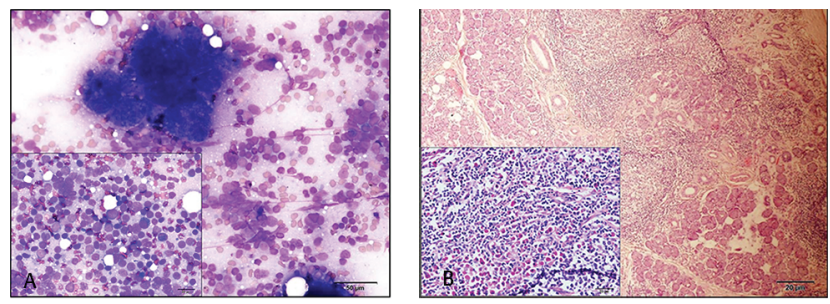

Fig. 1 (A) Photomicrograph depicting the fine-needle aspiration cytology of benign lymphoepithelial lesion showing polymorphous lymphoid infiltration along with scattered acini; May-Grunwald Giemsa 200×, inset 400×. (B) Corresponding tissue section showing benign lymphocytic infiltrate of salivary gland with parenchymal atrophy and lymphoepitheliotropism; Hematoxylin and Eosin 40x, inset $200 \times$.

is discussed herewith. A 59-year-old female presented with a right postauricular swelling (parotid), the initial FNAC report of which was inconclusive. Subsequently excision biopsy was done that misinterpreted it as LEC. IHC for pancytokeratin (PanCK; Dako) and leucocyte common antigen (LCA; Dako) were deemed to be positive in the atypical epithelial cells and the lymphoid cells, respectively. However, a review report was asked for on the same biopsy, which concluded lymphoepithelial sialadenitis with accompanying fibrosis as the final and consensus diagnosis, with the IHC being revised as PanCK positive in the remnant benign ductal cells, and LCA positive in the lymphoid cells ( - Fig. 4). The histiocytic population had been mistaken as poorly differentiated epithelial cells of LEC, destruction of the glandular and acinar architecture, and the accompanying infiltrating lymphoid cells further adding to the diagnostic confusion. The same patient again presented 2 years later with right submandibular swelling.

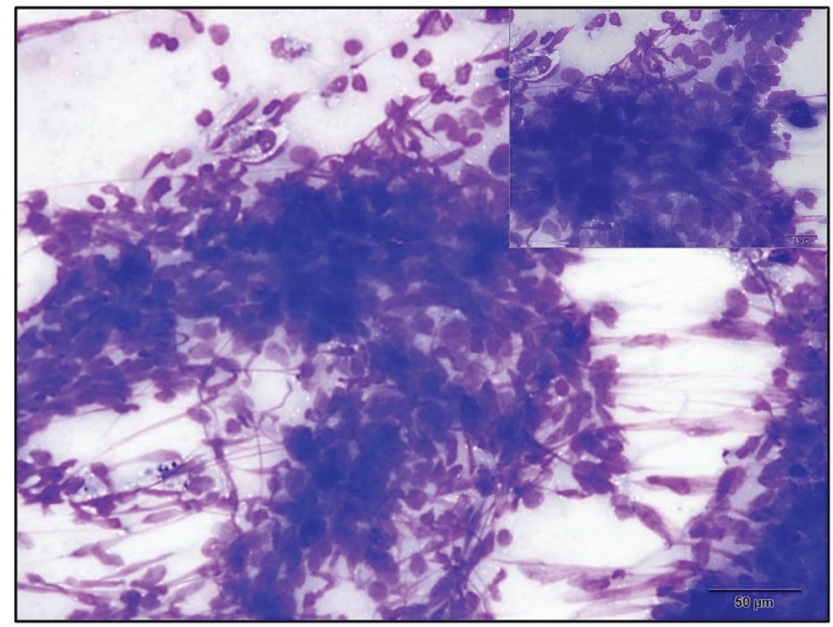

Fig. 2 Photomicrograph showing the fine-needle aspiration cytology for a case of lymphoepithelial carcinoma of the submandibular gland diagnosed on cytopathology as poorly differentiated malignancy (May-Grunwald Giemsa 200×, inset 400×).
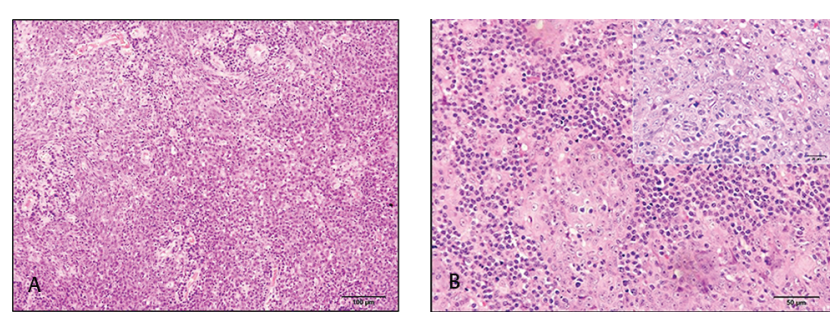

Fig. 3 (A) Photomicrograph exhibiting the corresponding tissue section of the case of lymphoepithelial carcinoma shown in Fig. 2, with (B) the presence of lymphoepithelial lesion and (inset) aggregates of poorly differentiated large polygonal epithelial cells ([A] 100×, [B] 200×, inset 400×; Hematoxylin and Eosin). 

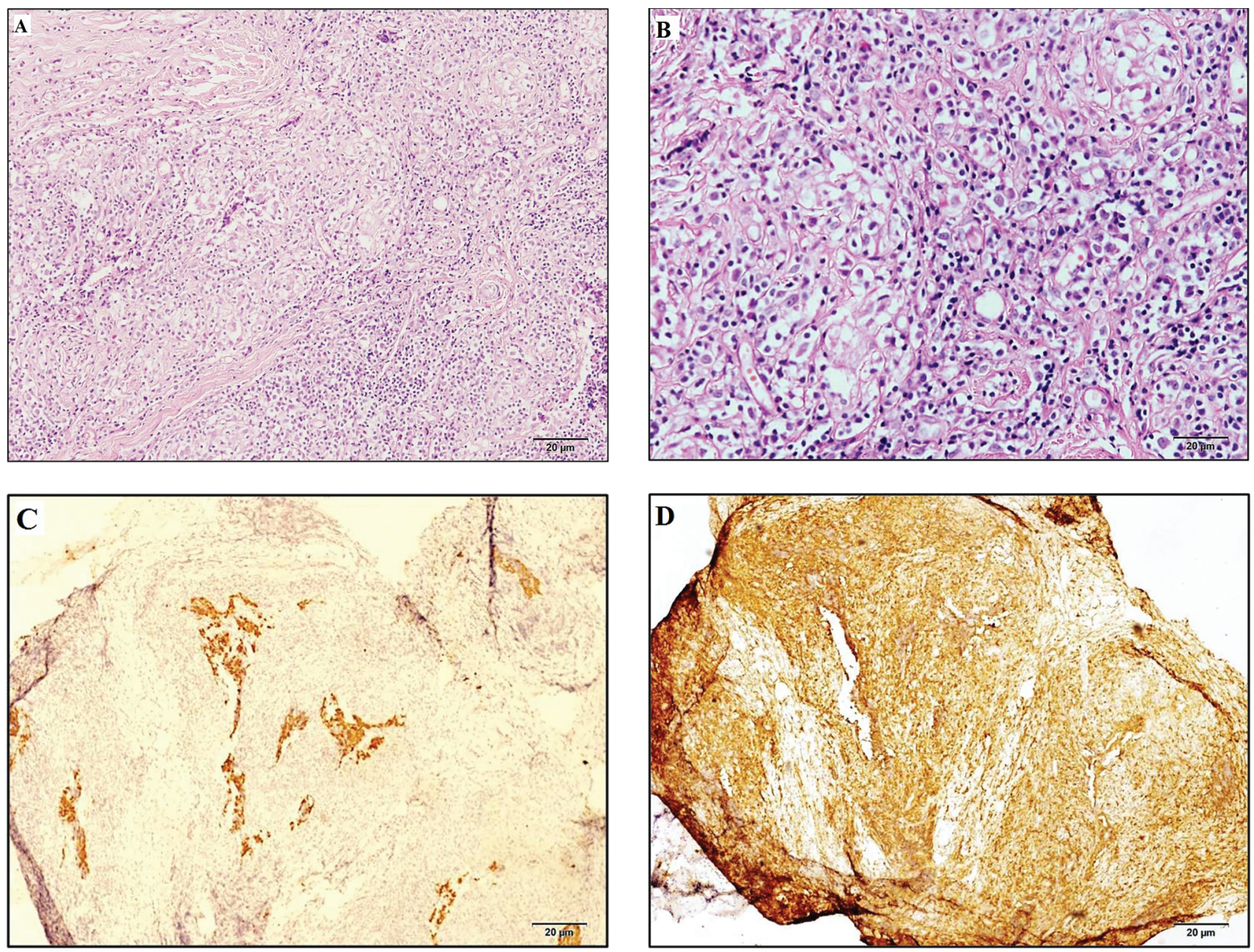

Fig. 4 Photomicrograph demonstrating the histopathological examination of lymphoepithelial sialadenitis of the parotid misinterpreted previously as lymphoepithelial carcinoma (Hematoxylin and Eosin [A] 100×, [B] 200×). Immunohistochemistry shows positivity of the remnant epithelial cells for PanCK (C), and the lymphoid cells for leucocyte common antigen (D) (C, D 100x).
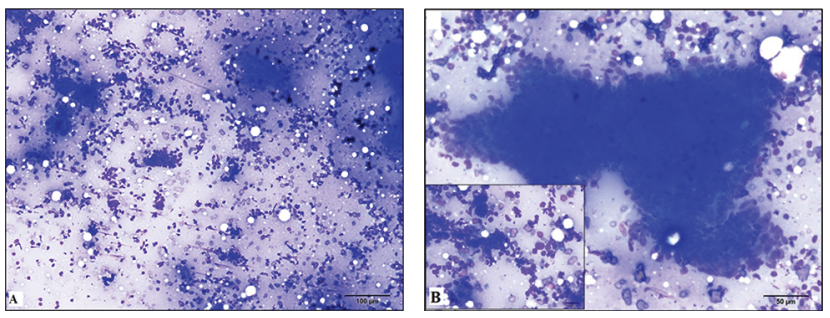

Fig. 5 Fine-needle aspiration cytology photomicrographs of the subsequent submandibular swelling of the patient mentioned in Fig. 4 misdiagnosed as lymphoepithelial carcinoma (May-Grunwald Giemsa [A] 100×, [B] 200×, inset 400×).

FNAC was done, which revealed a possibility of LEC ( - Fig. 5). The lesion was surgically excised. HPE, however, favored granulomatous lymphoepithelial sialadenitis with fibrosis (-Fig. 6). Tuberculosis as a primary etiology was ruled out though. As regards the association of LEC with Epstein-Barr virus (EBV), two cases showed positivity for EBV-latent membrane protein 1 (EBV-LMP1) IHC (BioGenex), while the other three cases were negative, thus accounting to an unlikely low proportion of positive cases (40\%).

\section{Discussion}

Lymphocytic infiltrates of the salivary glands are discerned in a spectrum of diseases, ranging from reactive to benign and malignant neoplasms. In many cases, the lymphocytic infiltrate is a minor inflammatory component that is easily distinguished from the primary disease processes. In some cases, however, the lymphocytic infiltrate emerges as the major component of the disease. Histopathologic features that distinguish reactive and benign lesions from malignant lesions are often subtle. ${ }^{2}$

Cases diagnosed as LELs encompassing benign LELs/lymphoepithelial sialadenitis/malignant LELs and as poorly differentiated malignancies were keenly analyzed. Lymphoepithelial sialadenitis is characterized by benign lymphocytic infiltrate of salivary gland with parenchymal atrophy and foci of ductal hyperplasia with lymphocytic epitheliotropism. The lobular architecture of the gland is usually preserved. In the early stages, the extent of lymphocytic infiltrate varies among the lobules of the gland, but in late stage disease, nearly all of the parenchyma is infiltrated with formation of lymphoid germinal centers. Multiple foci of 

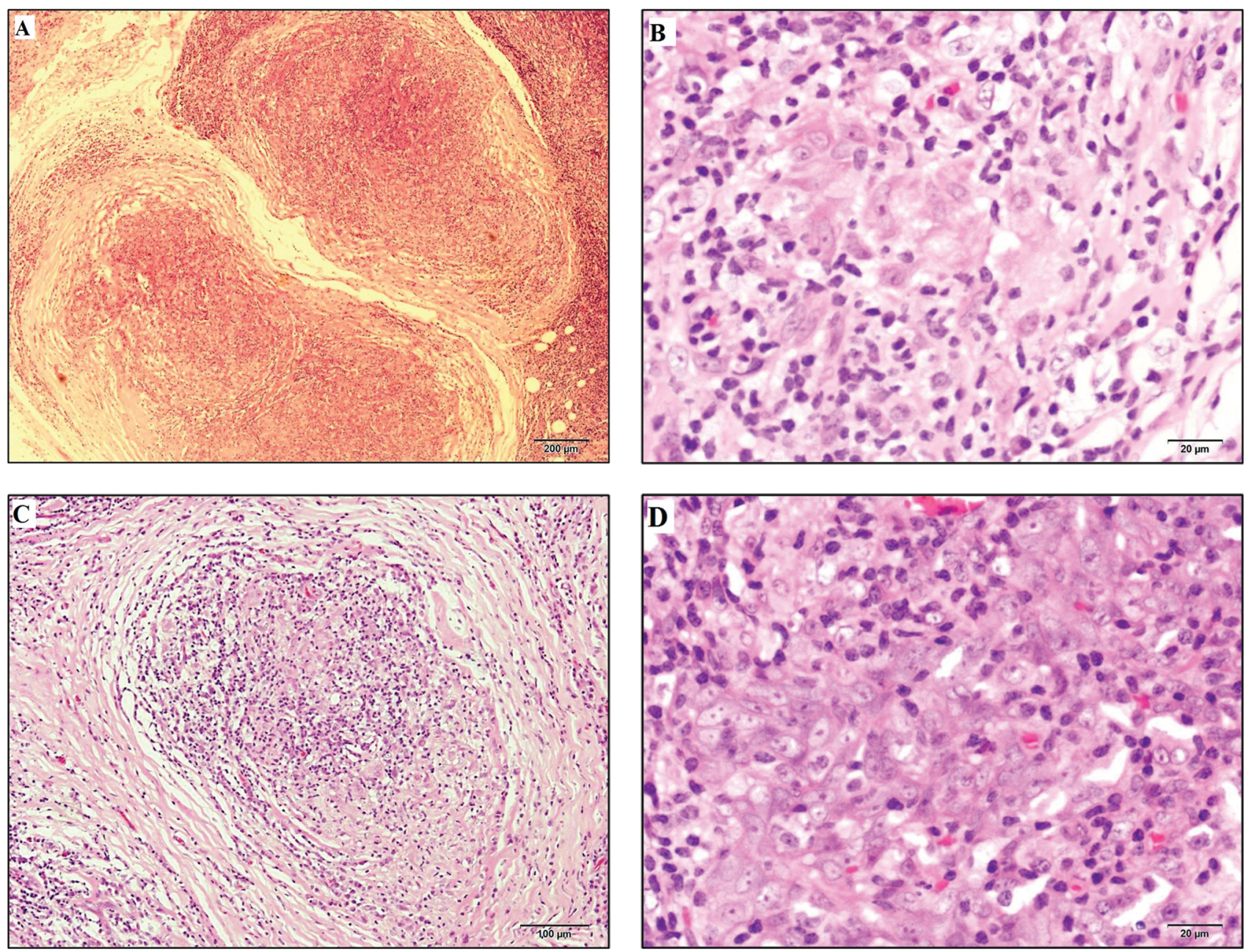

Fig. 6 Histopathological photomicrographs of the case shown in Fig. 5 conclusively diagnosed as granulomatous lymphoepithelial sialadenitis (Hematoxylin and Eosin [A] 40×, [B] 10×, [C] and [D] 400×).
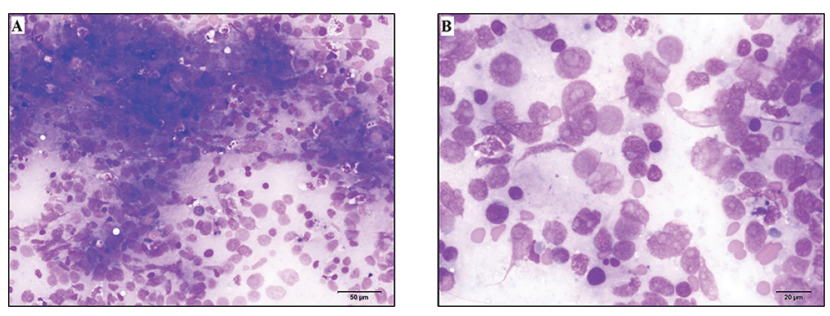

Fig. 7 Fine-needle aspiration cytology photomicrographs of a case of lymphoepithelial carcinoma misinterpreted as metastatic nasopharyngeal carcinoma (May-Grunwald Giemsa [A] 200×, [B] 400×).

ductal epithelial hyperplasia are permeated by lymphocytes, recognized as LELs. ${ }^{2-4}$

Biopsy was not warranted in most of the cases diagnosed as chronic sialadenitis. Two cases diagnosed cytopathologically as chronic sialadenitis of the parotid gland turned out to be benign salivary gland neoplasms on HPE-Warthin tumor and myoepithelioma. Warthin tumors have a variable population of lymphoid cells, which proved to be the diagnostic pitfall on FNAC. ${ }^{5-7}$ Ductal cells with oncocytic features were not observed in the cytology sample. Conversely, an inflammatory chronic sialadenitis that is lymphocytic rich may be
misinterpretedasWarthin tumordue to oncocyticmetaplasia. ${ }^{8}$ Myoepitheliomas can have a heterogenous appearance; subtypes of myoepitheliomas are classified by cell morphology: spindle (interlacing fascicles with a stroma-like appearance), plasmacytoid/hyaline (polygonal cells with eccentric nuclei and dense, nongranular or hyaline, abundant eosinophilic cytoplasm), epithelioid (nests or cords of round to polygonal cells, with centrally located nuclei and a variable amount of eosinophilic cytoplasm), and clear (polygonal cells with abundant optically clear cytoplasm, containing large amounts of glycogen but missing mucin or fat). ${ }^{9}$ The spindle-shaped cells of myoepithelioma along with the lymphoid population posed as the proverbial banana skin leading it to be misdiagnosed on FNAC as chronic sialadenitis with fibrosis in this study. ${ }^{10}$ Selective sampling is always an issue.

FNAC termed two cases of LECs, one arising from the minor salivary glands of the buccal mucosa and another originating from the parotid as metastatic poorly differentiated carcinomas. Thus, metastasis, especially from the nasopharyngeal carcinoma, can be confused with the primary tumor even if the lesion involves the parotid. LEC, a rare large cell undifferentiated carcinoma embedded within a dense lymphoid 

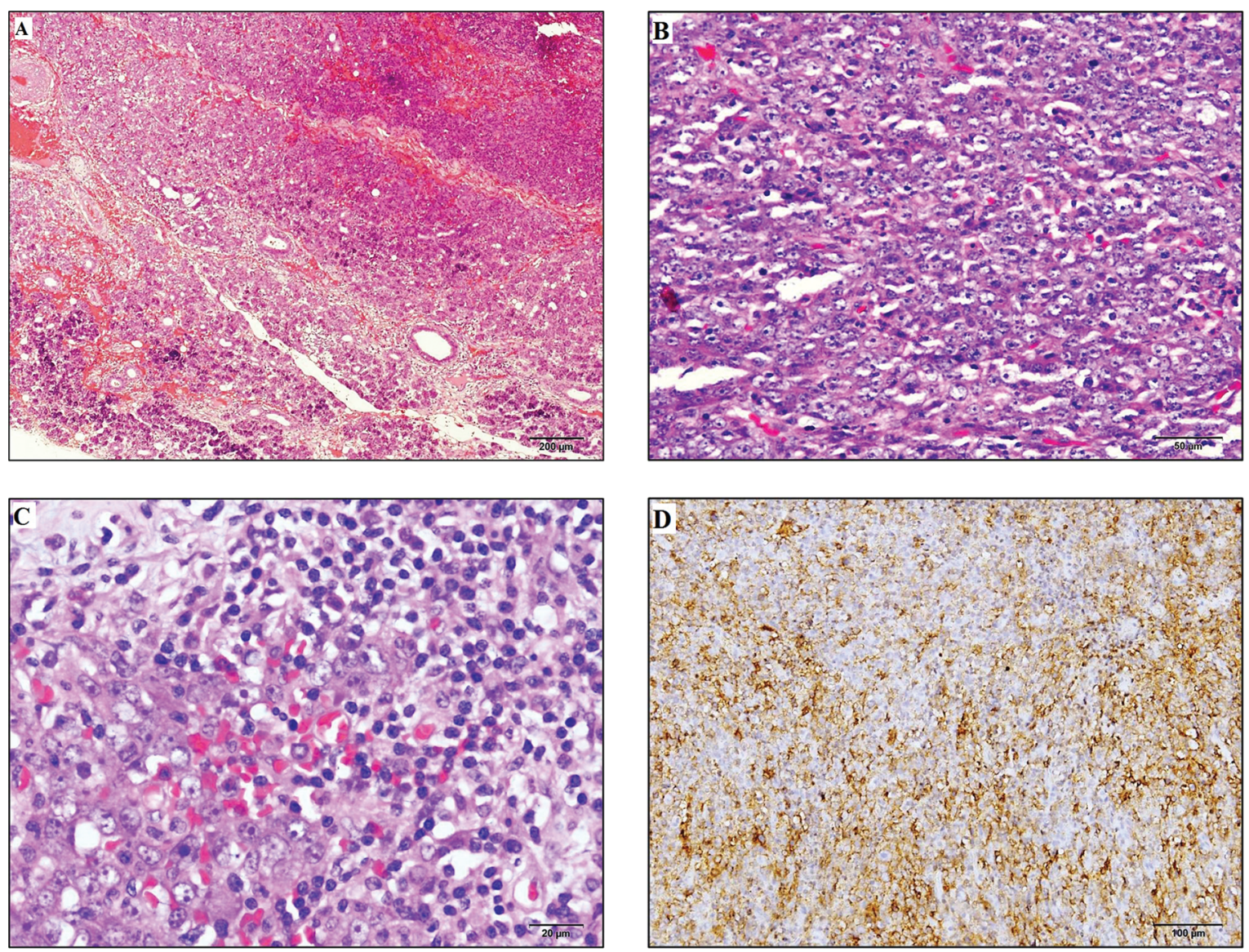

Fig. 8 Photomicrograph of the corresponding tissue section of the case of lymphoepithelial carcinoma mentioned in Fig. 7. Immunohistochemistry (IHC) for epithelial membrane antigen (EMA) highlighting the epithelial nature of the large atypical cells (Hematoxylin and Eosin [A] 40×, [B] 200×, [C] 400×; IHC for EMA [D] 100×).

stroma, constitutes only $0.4 \%$ of salivary gland neoplasms. The parotid gland is primarily affected, approximately $80 \%$ of cases, followed by the submandibular gland.,111 On cytopathology, the picture though can be heterogeneous. It may show a variable admixture of neoplastic epithelial cells intimately admixed with a variable population of lymphoid cells, or more commonly show a population of undifferentiated cells, which may lead to other differential diagnoses such as poorly differentiated malignancy/poorly differentiated carcinoma/metastatic carcinoma ( - Fig. 7). Conversely, the ductal cells of a benign process such as lymphoepithelial sialadenitis or granulomatous lesion may look so atypical, so as to mimic LEC, and accordingly, a granulomatous entity should also be kept in the differential diagnoses. The histiocytic population may simulate neoplastic epithelial cells, as was the scenario in the previously mentioned case of the 59-year-old female. Thus, caution should be exercised not to misconstrue the histiocytic cells of granulomatous sialadenitis as that of poorly differentiated epithelial lineage. Destruction of the salivary gland architecture as seen in LECs is a morphologic feature in granulomatous/nongranulomatous lymphoepithelial sialadenitis, as well as the infiltrating lymphoid population. IHC should be interpreted appropriately correlating with the morphology and should not be regarded as the savior to unraveling the diagnostic dilemma in such situations. Lymphoepithelial sialadenitis, although it shares the prominent lymphoid population with LEC, lacks cytologic features of malignancy, desmoplastic stroma, invasion of adjacent tissues, and EBV association. ${ }^{2}$ Meticulous HPE is the sine qua non to solving such a diagnostic quandary.

As mentioned, metastatic carcinoma is another potential pitfall while dealing with LECs. Presence of the infiltrating significant lymphoid population and/or associated BLEL or lymphoepithelial nests similar to BLEL are helpful clues in differentiating LECs from poorly differentiated malignancy/carcinoma and metastatic carcinoma, usually from the nasopharynx ( $\mathbf{- F i g .}$. 8). In fact, the presence of accompanying BLEL is the most important finding in establishing the histologic diagnosis of LEC. If, however, BLELs are absent, distinguishing LECs from metastatic undifferentiated nasopharyngeal carcinoma can be quite challenging on morphology as the two entities share similar cytologic, architectural, and immunohistochemical features. Moreover, ethnic predilection and association with EBV infections are other common threads between these two. ${ }^{1,2}$ Fortunately though, the parotid gland is the predominant site of occurrence of LEC and an infrequent site of metastasis from nasopharyngeal carcinoma, which more typically metastasizes to the cervical or submandibular lymph nodes. IHC does not offer much additional help in such cases. Careful clinical assessment and 
thorough evaluation of the nasopharynx and Waldeyer's ring region are essential to exclude the possibility of metastatic disease (which is more common), and before accepting the salivary gland tumor as primary LEC. ${ }^{1,11}$

IHC though helps differentiate LECs from other metastatic malignancies like amelanotic melanoma or other carcinomas, and large cell lymphoid and histiocytic neoplasms. ${ }^{12}$ Large cell undifferentiated carcinoma, which lacks histomorphologic features of either glandular or epidermoid differentiation, is another differential diagnosis of LEC. These are typically reactive for cytokeratins, but the prominent lymphoid component of LEC as has been mentioned above again comes to the rescue in resolving this conundrum, being absent in large cell undifferentiated carcinoma. ${ }^{13}$ Not only this, the lymphoid stroma is also instrumental in limiting the aggressiveness of LEC, perhaps contributing to the better prognosis of this carcinoma than the other undifferentiated carcinomas of the salivary glands. ${ }^{1}$

The near $100 \%$ association of EBV with salivary gland LEC from the endemic areas and the presence of the virus in a clonal episomal form suggest an important role of EBV in tumourigenesis. ${ }^{11,14-16}$ In our study, however, IHC detected expression of EBV-LMP1 in the tumor cells in only two out of five cases of LEC (40\%). In-situ hybridization is more sensitive in proving this association, by detection of EBV-encoded RNA and EBV-DNA in the tumor cells. IHC expression of EBV-LMP1 is more variable, thus clarifying the reason for the negativity of EBV-LMP1 in the three cases, and the unlikely low association in an otherwise endemic area. In patients from nonendemic areas, EBV is usually absent, although rare cases may harbor the virus. These findings indicate complex interactions of ethnic, geographic, and viral factors in the pathogenesis of salivary gland LEC. ${ }^{11,14-17}$ LECs have a strong tendency to metastasize to the regional cervical lymph nodes, and approximately 20\% develop distant metastasis, most commonly to the lung, liver, bone, and brain. However, 75 to $86 \%$ of patients are documented to survive when treated with surgery, including neck dissection, and radiation therapy, although local recurrence can occur. ${ }^{11,15,16,18}$

\section{Conclusion}

LELs of salivary glands can pop up as a pathologist's quagmire at times. This study tried to highlight the diagnostic issues related to these lesions, both from a cytopathologic and a histopathologic perspective. LECs can mimic undifferentiated carcinoma, metastatic carcinoma, or even reactive/granulomatous lesions not only on cytology but also on HPE of the excised lesion. Even benign salivary gland tumors can be masked by chronic sialadenitis/LELs. Careful attention to pathologic detail and clinical inputs is the cornerstone in arriving at the correct diagnosis, thus paving the way for appropriate management.

\section{Authors' Contribution}

Zachariah Chowdhury was involved in concepts, design, definition of intellectual content, literature search, clinical studies, experimental studies, data acquisition, data analysis, statistical analysis, manuscript preparation, manuscript editing, and manuscript review. Vandana Raphael was involved in concepts, design, definition of intellectual content, literature search, data acquisition, data analysis, statistical analysis, manuscript preparation, manuscript editing, and manuscript review. Yookarin Khonglah was involved in concepts, design, definition of intellectual content, literature search, data acquisition, data analysis, statistical analysis, manuscript preparation, manuscript editing, and manuscript review. Jaya Mishra was involved in concepts, design, definition of intellectual content, data acquisition, data analysis, manuscript preparation, manuscript editing, and manuscript review. Evarisalin Marbaniang was involved in concepts, design, definition of intellectual content, data acquisition, data analysis, manuscript preparation, manuscript editing, and manuscript review. Biswajit Dey was involved in concepts, design, definition of intellectual content, data acquisition, data analysis, manuscript preparation, manuscript editing, and manuscript review. Zachariah Chowdhury and Vandana Raphael have provided guarantee for this manuscript.

\section{Statement of Ethics}

The study followed all the guidelines of the institutional ethics committee and was performed with appropriate participants' informed consent in compliance with the World Medical Association Declaration of Helsinki.

\section{Conflict of Interest}

The authors declare that no conflicts of interest and no sponsorship or funding were received for this research.

\section{Acknowledgments}

The authors acknowledge the help of Neizekhotuo Brian Shunyu, MS, Associate Professor, Department of Otorhinolaryngology, North Eastern Indira Gandhi Regional Institute of Health \& Medical Sciences, Shillong, India, for guiding us through the work.

\section{References}

1 Schneider M, Rizzardi C. Lymphoepithelial carcinoma of the parotid glands and its relationship with benign lymphoepithelial lesions. Arch Pathol Lab Med 2008;132(2):278-282

2 Ellis GL. Lymphoid lesions of salivary glands: malignant and benign. Med Oral Patol Oral Cir Bucal 2007;12(7):E479-E485

3 Ihrler S, Zietz C, Sendelhofert A, Menauer F, Blasenbreu-Vogt S, Löhrs U. [Differential diagnosis of lymphoepithelial lesions of salivary glands. With particular reference to characteristic duct lesions]. Pathologe 2000;21(6):424-432

4 Ihrler S, Zietz C, Sendelhofert A, Riederer A, Löhrs U. Lymphoepithelial duct lesions in Sjögren-type sialadenitis. Virchows Arch 1999;434(4):315-323

5 Sandhu VK, Sharma U, Singh N, Puri A. Cytological spectrum of salivary gland lesions and their correlation with epidemiological parameters. J Oral Maxillofac Pathol 2017;21(2):203-210

6 Das DK, Petkar MA, Al-Mane NM, Sheikh ZA, Mallik MK, Anim JT. Role of fine needle aspiration cytology in the diagnosis of swellings in the salivary gland regions: a study of 712 cases. Med Princ Pract 2004;13(2):95-106 
7 Mukundapai M, Sharma N, Patil A, Gopal C. Fine-needle aspiration cytology of salivary gland lesions: a revised classification based on "Milan system"-4years experience of tertiary care cancer center of south India. J Cytol 2020;37(1):12-17

8 Salehi S, Maleki Z. Diagnostic challenges and problem cases in salivary gland cytology: a 20-year experience. Cancer Cytopathol 2018;126(2):101-111

9 Weitzel M, Cohn JE, Spector H. Myoepithelioma of the parotid gland: a case report with review of the literature and classic histopathology. Case Rep Otolaryngol 2017;2017:6036179

10 Ahn S, Kim Y, Oh YL, Oh YL. Fine needle aspiration cytology of benign salivary gland tumors with myoepithelial cell participation: an institutional experience of 575 cases. Acta Cytol 2013;57(6):567-574

11 Tsang WY, Kuo TT, Chan JKC, Lymphoepithelial carcinoma. In: Barnes L, Eveson JW, Reichart P, Sidransky D, eds. World Health Organization Classification of Tumours. Pathology and Genetics of Head and Neck Tumours. Lyon: IARC Press; 2005 251-252

12 Ellis GL, Auclair PL, Tumors of the Salivary Glands: Atlas of Tumor Pathology, 3rd series, fascicle 17. Washington, DC: Armed Forces Institute of Pathology; 1996: 311-318, 411-413
13 Sheen TS, Tsai CC, Ko JY, Chang YL, Hsu MM. Undifferentiated carcinoma of the major salivary glands. Cancer 1997;80(3):357-363

14 Hamilton-Dutoit SJ, Therkildsen $\mathrm{MH}$, Neilsen $\mathrm{NH}$, Jensen $\mathrm{H}$, Hansen JP, Pallesen G. Undifferentiated carcinoma of the salivary gland in Greenlandic Eskimos: demonstration of Epstein-Barr virus DNA by in situ nucleic acid hybridization. Hum Pathol 1991;22(8):811-815

15 Leung SY, Chung LP, Yuen ST, Ho CM, Wong MP, Chan SY. Lymphoepithelial carcinoma of the salivary gland: in situ detection of Epstein-Barr virus. J Clin Pathol 1995;48(11):1022-1027

16 Tsai CC, Chen CL, Hsu HC. Expression of Epstein-Barr virus in carcinomas of major salivary glands: a strong association with lymphoepithelioma-like carcinoma. Hum Pathol 1996;27(3):258-262

17 Gallo O, Santucci M, Calzolari A, Storchi OF. Epstein-Barr virus (EBV) infection and undifferentiated carcinoma of the parotid gland in Caucasian patients. Acta Otolaryngol 1994;114(5):572-575

18 Kuo T, Hsueh C. Lymphoepithelioma-like salivary gland carcinoma in Taiwan: a clinicopathological study of nine cases demonstrating a strong association with Epstein-Barr virus. Histopathology 1997;31(1):75-82 